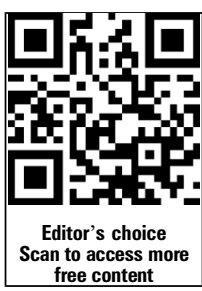

${ }^{1}$ Prince of Wales Clinical School, University of New South Wales, Randwick, New South Wales, Australia ${ }^{2}$ Brain and Mind Research Institute, The University of Sydney, Camperdown, New South Wales, Australia ${ }^{3}$ Department of Neurosurgery, Mayo Clinic, Rochester, Minnesota, USA

${ }^{4}$ Department of Neurosurgery, Louisiana State University Health Science Center, New Orleans, Louisiana, USA ${ }^{5}$ Department of Neurological Surgery, Northwestern Feinberg School of Medicine, Chicago, Illinois, USA

\section{Correspondence to} Dr Neil G Simon, Prince of Wales Clinical School, University of New South Wales, Barker St, Randwick, NSW 2031, Australia; n.simon@unsw.edu.au

Received 1 January 2015 Revised 12 March 2015 Accepted 3 April 2015 Published Online First 28 April 2015

\section{SLinked}

- http://dx.doi.org/10.1136/ jnnp-2015-310420

\section{CrossMark}

To cite: Simon NG, Spinner RJ, Kline DG, et al. J Neurol Neurosurg Psychiatry 2016:87:198-208.

\title{
Advances in the neurological and neurosurgical management of peripheral nerve trauma
}

\author{
Neil G Simon, ${ }^{1,2}$ Robert J Spinner, ${ }^{3}$ David G Kline, ${ }^{4}$ Michel Kliot $^{5}$
}

\begin{abstract}
Peripheral nerve trauma frequently affects younger people and may result in significant and long-lasting functional disability. Currently, diagnosis and monitoring of peripheral nerve injury relies on clinical and electrodiagnostic information, supplemented by intraoperative electrophysiological studies. However, in a significant proportion of nerve injuries, the likelihood of spontaneous regeneration resulting in good functional outcome remains uncertain and unnecessary delays to treatment may be faced while monitoring for recovery. Advances in non-invasive imaging techniques to diagnose and monitor nerve injury and regeneration are being developed, and have the potential to streamline the decision-making process. In addition, advances in operative and non-operative treatment strategies may provide more effective ways to maximise functional outcomes following severe peripheral nerve trauma. This review discusses these advances in light of the current state of the art of management of peripheral nerve trauma.
\end{abstract}

\section{INTRODUCTION}

Peripheral nerve trauma (PNT) is a major source of disability in the community. Annual incidence is approximately 45 cases per 100000 , similar to the incidence of epilepsy. ${ }^{1}$ Severe PNT complicates approximately $5 \%$ of polytrauma cases, such as motor vehicle accidents. ${ }^{2}$ The implications of PNT can be particularly significant as it commonly affects younger people of working age and may result in impaired limb function, and reduced ability to participate in the workforce. ${ }^{3} 4$ Functional outcomes vary depending on the nature of the injury and with appropriate treatment, may range from being excellent in patients with transection of a single upper limb nerve to very poor in patients with complex brachial plexus trauma with nerve root avulsion. ${ }^{5-7}$

Despite extensive clinical and surgical experiences, there remain limitations in the diagnosis and management of PNT, including early determination of nerve injuries that require surgical intervention, poor functional recovery in muscles distant from the site of nerve trauma, prevention and management of painful neuroma formation, and incomplete functional improvement despite successful reinnervation. Recent advances in diagnostic and management techniques may help overcome a number of these issues. The present review will discuss developments in multimodality assessment of PNT, including operative electrical assessment, emerging imaging techniques with the potential to detect early peripheral nerve regeneration, and novel surgical and medical therapeutic approaches to optimise functional recovery.

\section{GRADING NERVE INJURIES}

The first step in determining the appropriate management of PNT is to identify the extent of the nerve injury. Two major grading classification systems have been separately proposed by Seddon ${ }^{8}$ and Sunderland. ${ }^{9}$ Seddon's classification system is the simplest, with three types of nerve injury, neurapraxia, axonotmesis (figure 1) and neurotmesis, based on the degree of histological damage to the nerve. Sunderland's system provides more detailed classification of nerve injuries. Neurapraxia is classified as grade I injury. Grade II injuries involve damage to the axon, but the endoneurium remains intact. In grade III injuries, the axon and endoneurium are severed; grade III injuries may also include a mix of isolated axon and endoneurial damage, and more extensive connective tissue injury. Grade IV injuries involve injury to the axon, endoneurial tubes, perineurium and fascicular structures, but the epineurium remains intact. In grade V injuries, there is complete transection of all elements of the nerve, including the epineurium. At least $70 \%$ of serious injuries leave the peripheral nerve in continuity even when nerve connective tissue elements have been disrupted.

\section{Implications for nerve regeneration and functional recovery}

Neuropraxic injuries usually recover within 212 weeks of the injury from the restoration of the myelin sheath. Very mild neurapraxia may reverse in hours, which reflects transient metabolic derangement within the nerve rather than demyelination.

When axonal injury has occurred, sprouts emerge from axons at the proximal stump and grow distally at a rate of $1-3 \mathrm{~mm}$ per day. ${ }^{9}$ In partial injuries, where there are undamaged axons innervating the muscle, terminal sprouts from these preserved axons extend to denervated muscle endplates. Axonotmetic injuries typically recover some function without surgical intervention, with the time course of recovery partly determined by the extent of axonal injury and the distance between the site of nerve injury and the affected muscle.

Without surgical approximation of viable nerve stumps, or resection and then repair of neurotmetic lesions in continuity, recovery from neurotmetic injuries is limited. However, it should be noted that these lesions in continuity that convey impulses on 
Figure 1 Histological, electromyography (EMG) and diffusion tensor tractography (DTT) changes following severe axonotmetic injury. Wallerian degeneration of distal axons starts soon after nerve injury and macrophages clear nerve debris (1 week). Bands of Büngner act as guides for regenerating axonal sprouts originating from the proximal nerve stump (1 month). For a nerve injury $10 \mathrm{~cm}$ from the target, regenerating axons may be expected to reach the target muscle by about 4 months. In terms of EMG findings (middle panel), on the day of nerve injury, EMG will not demonstrate any motor unit potentials and this situation will remain until regenerating axons have reached the target muscle, when 'nascent' motor units will be seen (4 months). Operative compound nerve action potential recordings or perhaps novel DTT techniques (bottom panel, adapted from ${ }^{37}$ with permission from Lippincott Williams and Wilkins/ Wolters Kluwer Health) may detect axon regeneration earlier, when regenerating axon sprouts are advancing but have not yet reached their target muscle.
1 day

1 week

Nerve Histology
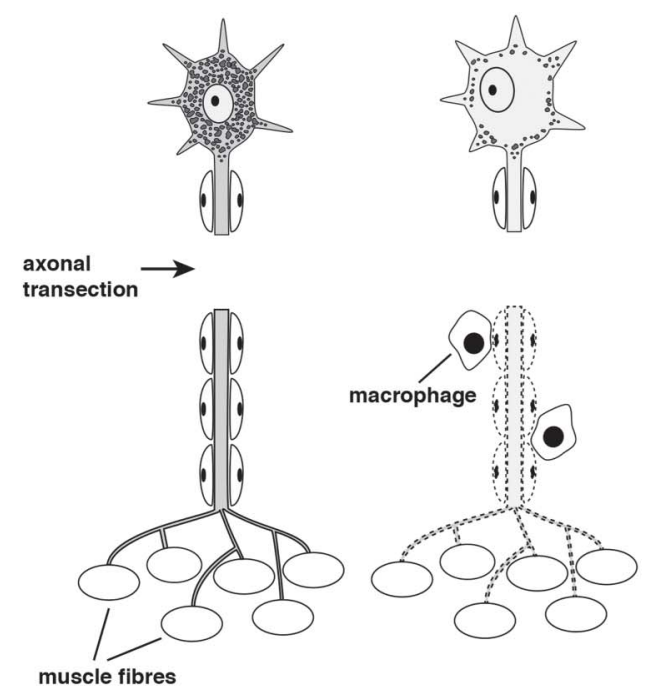

EMG

no motor units

no motor units

no motor units

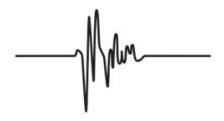

nascent motor units

4 months

1 month
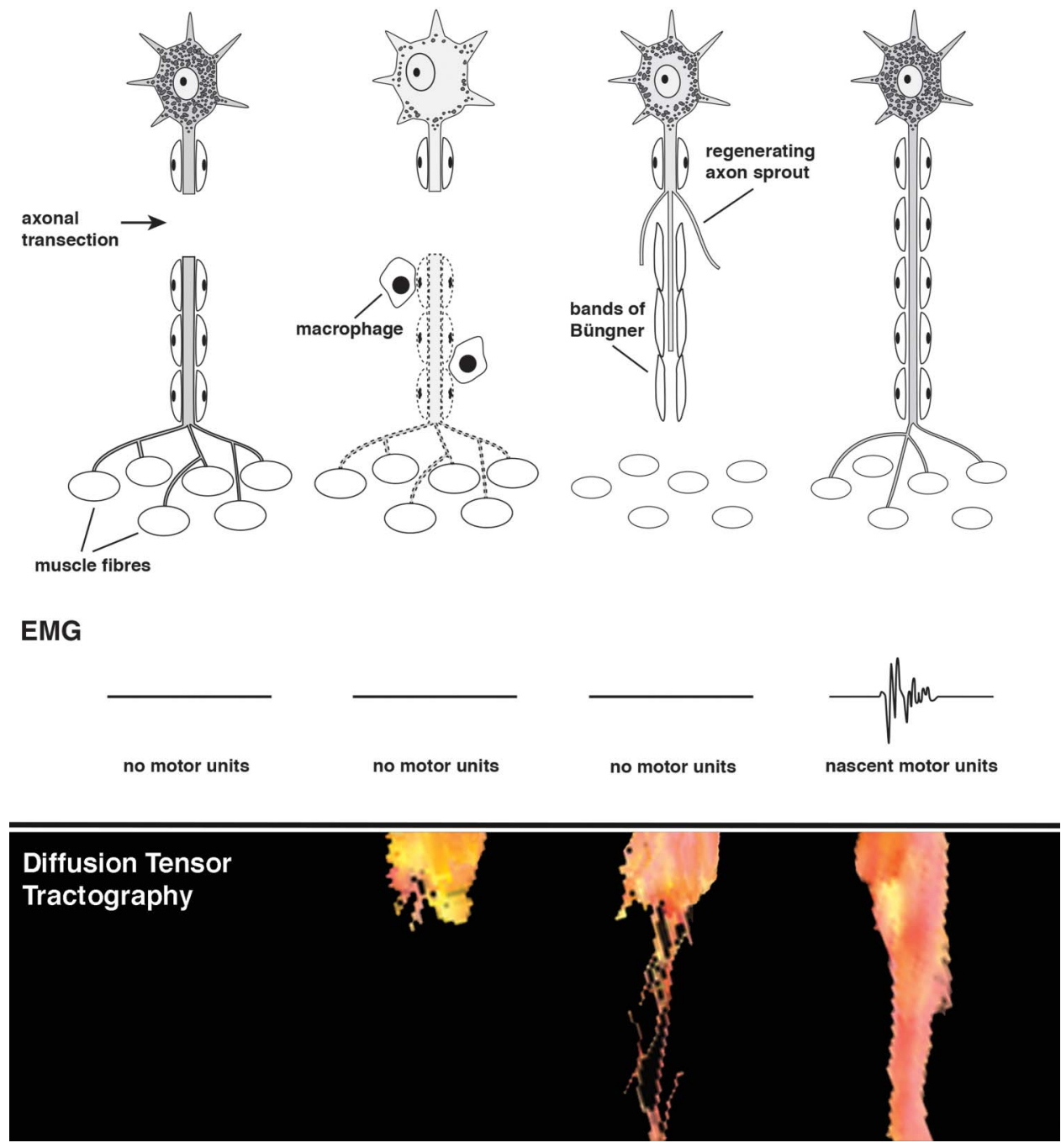

intraoperative electrophysiological testing are likely to undergo some spontaneous recovery.

\section{SELECTING PATIENTS FOR INTERVENTION}

The timing of interventions following PNT is critical. Earlier surgical intervention results in the best outcome in those patients with neurotmetic nerve injuries, where spontaneous regeneration is unlikely. Conversely, better outcomes may be observed from spontaneous axonal regeneration and muscle reinnervation processes in patients with axonotmetic injuries and in many Sunderland grade III injuries, where operative electrophysiological studies suggest that axonal regeneration through the injured segment is likely. Thus, patient selection is critically important when deciding the appropriate treatment strategy (figure 2).

\section{Electrodiagnostic evaluation after peripheral nerve injury}

Nerve conduction studies (NCS) can identify evidence of conduction block or slowing, consistent with demyelination (neurapraxia), and reduced compound muscle action potential (CMAP) amplitude suggestive of axonal injury. Electromyography (EMG) may identify fibrillations and positive sharp waves, arising from denervated muscle fibres, suggesting axonal injury. Rearrangement of motor units (MUs) accompanies reinnervation, consisting of MU polyphasia and prolonged duration in the earlier phases indicating inefficient and desynchronised conduction between the muscle fibres innervated by a single axon (figure 1). Later in the course of reinnervation, MUs may become of high amplitude, when individual muscle fibre action potentials are more synchronised and hence summate to produce a taller potential.

A commonly held view is that electrodiagnostic studies should be deferred for up to 3 weeks after nerve injury to allow for the development of fibrillations that give an indication of the extent of nerve injury. However, this ignores the valuable information that may be identified on NCS and EMG much sooner than this. Early studies may demonstrate features that enable distinction between the different grades of nerve injury despite fibrillations not necessarily having developed. Wallerian degeneration following axonal injury starts within days of injury and is often complete within a week. As such, the amplitude of the CMAP elicited with distal nerve stimulation will give an indication of whether axonal injury has occurred. Second, MU recruitment on EMG studies will 


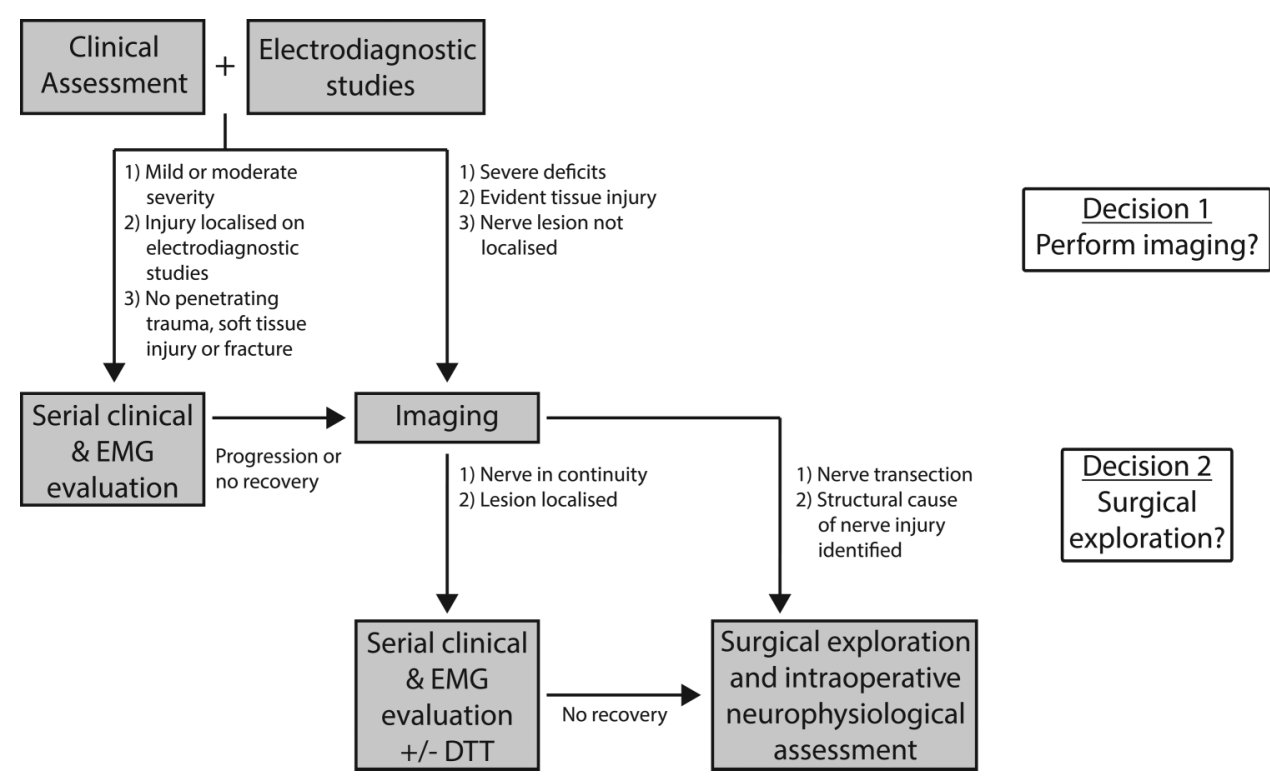

Figure 2 Flow chart detailing a suggested diagnostic and decision-making process in patients with nerve trauma. EMG, electromyography; DTT, diffusion tensor tractography.

distinguish between a neurotmetic or severe axonotmetic injury and neurapraxia or more mild axonotmetic injuries. In the former category, no MUs will be activated with voluntary effort, while in the latter category, reduced recruitment of normal MUs will be seen very early in the course of injury. EMG in severe neuropraxic injuries may also not identify MUs with effort, but in this instance motor NCS should be able to distinguish neurapraxia from severe axonal injury.

Unfortunately, electrodiagnostic studies are an imperfect means with which to grade nerve injuries. Distal responses on motor NCS may remain present for 2-3 days after complete nerve transection and be mistaken for severe conduction block and hence, neurapraxia when stimulation of the nerve proximal to the injury does not evoke a muscle response. The absence of MUs on EMG during voluntary activation of a muscle does not distinguish between a severe axonotmetic injury and a neurotmetic injury, and interpretation of this finding may be further complicated by the presence of concomitant upper motor neuron injury or focal nerve demyelination in more complex trauma.
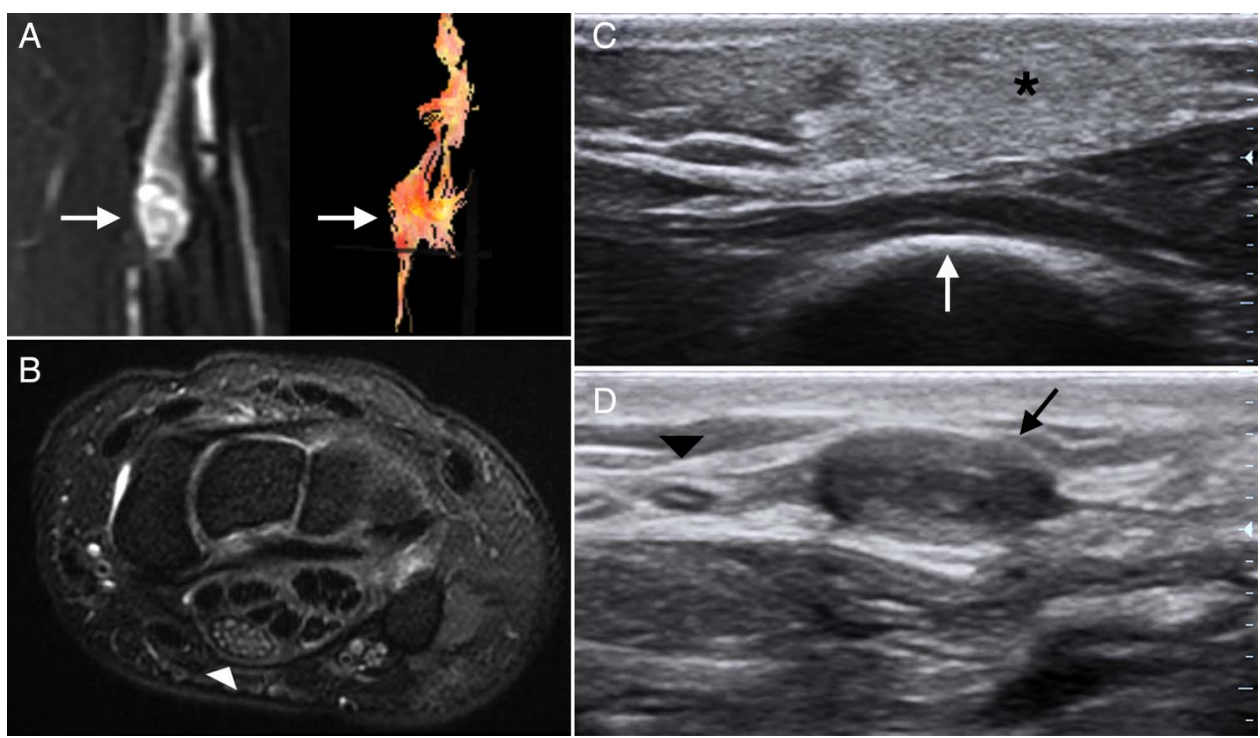

Figure 3 MR neurography and ultrasound findings in peripheral nerve trauma. Near total transection of the median nerve in the arm is demonstrated on fat-suppressed T2-weighted imaging and diffusion tensor tractography (DTT) (A). On T2-weighted images the nerve terminates at an enlarged, T2-hyperintense stump suggesting traumatic neuroma ( $\mathrm{A}$, left panel, arrow). Trackable fibres largely terminate at the proximal stump on DTT studies (A, right panel, arrow). Median nerve enlargement and hyperintensity at the wrist is demonstrated on fat-suppressed T2-weighted images in a patient with carpal tunnel syndrome ( $B$, white arrowhead). In an infant with perinatal wrist drop, ultrasound studies identified enlargement of the radial nerve in the spiral groove (white arrow) with loss of normal fat architecture overlying the nerve $\left({ }^{*}\right)$ suggesting fat necrosis and nerve injury secondary to extrinsic pressure. Ultrasound studies identified features of a traumatic sural neuroma in a patient following a posterior leg laceration (D). A normal sural nerve fascicle is demonstrated (black arrowhead), lying next to the enlarged hypoechoic neuroma (black arrow). 
Imaging assessment of PNT

The past two decades have seen a rapid expansion in the ability to image the peripheral nervous system. Parallel advances in MRI and ultrasound technology have added these two complimentary techniques to the diagnostic toolkit of PNT. Imaging has allowed for more detailed analysis of the extent of nerve and soft tissue injury, permitting the development of more comprehensive treatment plans and potentially obviating the need for exploratory surgery. In addition, imaging may be able to predict those patients for whom early intervention is important and those for whom an observational approach is appropriate.

Incorporating nerve imaging modalities in the initial work-up of patients with nerve injury may delineate the anatomic context of the nerve injury, such as aberrant structures contributing to nerve trauma. For example, a bony fragment following orthopaedic injury may be seen to distort the course of the nerve. ${ }^{10}$ An intraneural ganglion cyst may be detected in a patient with peroneal neuropathy. ${ }^{11} 12$ The extent of nerve injury may also be determined (figure 3 ). Imaging may identify nerve transection with discontinuity between stump ends of the nerve. ${ }^{13}$ Identifying these features will modify treatment considerations.

Demonstrating a traumatic neuroma on imaging suggests severe nerve injury (figure 3). Traumatic neuromas are formed when attempts at regeneration are unsuccessful, and instead a collection of connective tissue and axonal elements forms. ${ }^{14}$ A neuroma will develop at the stump of a transected nerve and appears as a bulbous expansion. ${ }^{15}$ Severe nerve traction may also produce a neuroma-in-continuity, which appears as an ovoid expansion within the nerve and is associated with severe axonal injury, although successful regeneration may still be possible without surgical intervention. It should be pointed out that identifying fascicular continuity through a lesion in continuity on imaging could be promising for recovery, but does not guarantee it.

Imaging of muscle with either MRI or ultrasound provides additional information regarding the extent of denervation and the patterns of muscles involved, and may be a useful adjunct to electrodiagnostic studies in some situations. ${ }^{16}{ }^{17}$ Muscle imaging changes have been shown to be the earliest positive sign of muscle denervation, before the detection of fibrillations on EMG studies.

\section{MR neurography}

MR neurography (MRN) is frequently performed in patients with PNT. While there is no formal consensus as to what constitutes MRN, multiplanar high-resolution two-dimensional T1 and fat-suppressed heavily T2-weighted sequences are fundamental to almost every MRN study. The T1 sequences are useful to delineate the anatomical context of the nerve, to outline the epineurial fat plane, to determine nerve size and to identify the fascicular structure of the nerve. Fat-saturated T2-weighted sequences detect increased water content reflecting oedema of the injured nerve.

There are several common findings on MRN in various nerve injuries. Specifically, peripheral nerves become larger and may lose fascicular definition. Nerves may also become hyperintense on T2-weighted sequences (figure 3). Finally, an injured nerve segment may demonstrate contrast enhancement, although this is variable and not a reliable indicator of nerve trauma. ${ }^{18}$ To date, MRN has failed to differentiate distal stump degeneration mixed with regenerating axons in nerves that do not recover from those that do recover.
Nerve ultrasound

Advances in transducer technology, the introduction of highfrequency probes up to $22 \mathrm{MHz}$, and improvements in image postprocessing have enabled bedside ultrasound imaging of even the smallest peripheral nerves. Strengths of ultrasound imaging include its portability and tolerability. Of further benefit, ultrasound allows dynamic imaging and as such may demonstrate changes in the nerve in its anatomical context with limb movement, which may be relevant when considering the mechanism of nerve injury. Like MRN, ultrasound demonstrates increased nerve calibre and loss of the normal fascicular pattern following nerve injury. In addition, echogenicity may be reduced, analogous to changes in T2 nerve intensity on MRN (figure 3). ${ }^{19-21}$

Ultrasound is complimentary to other clinical and electrodiagnostic techniques for nerve injury and is, therefore, being increasingly adopted in these settings. ${ }^{13} 22$ Besides use in the clinic, a number of other potential roles of nerve ultrasound are evolving. Intraoperative nerve ultrasound is being developed and changes of nerve injury correlate well with intraoperative electrophysiological monitoring, nerve dissection and histopathological findings in resected nerve segments. ${ }^{23}$ An additional role of ultrasound, that is yet to be fully explored, is its use in guiding electrodiagnostic studies. Ultrasound may improve the performance of more difficult conventional NCS by identifying the location of the nerve. ${ }^{24}$ Ultrasound-guided, percutaneous, near-nerve stimulation and recording warrants further exploration, and may obviate exploratory surgery and intraoperative neurophysiological mapping in some patients.

Limitations of ultrasound include diminished resolution of nerves located deeply (such as the proximal sciatic nerve in patients with extensive subcutaneous tissue) or underneath bone. Ultrasound image acquisition is also operator dependent and successful studies require substantial understanding of anatomy and technical experience.

\section{MONITORING RECOVERY}

The necessary course of treatment for PNT may be readily apparent after initial clinical, electrodiagnostic and imaging evaluations. For example, acute, clean transection of a single peripheral nerve, such as that following a sharp penetrating injury, requires prompt surgical neurorrhaphy obviating the need for serial observation. Similarly, early repair of blunt nerve transection is indicated, but after a $2-3$-week delay to allow the viable portions of the nerve stumps to be delineated. ${ }^{25}$ However, there remains a substantial proportion of patients with lesions in continuity, where the nature of the nerve injury and hence, the prognosis and most appropriate intervention remains uncertain. In these patients there are two possible approaches: a 'waiting game' of serial clinical and electrodiagnostic assessments (approximately every 6-8 weeks) looking for clinical and electrophysiological evidence of muscle reinnervation or operative exploration, with intraoperative electrophysiological assessment of nerve regeneration to determine the need for resection and repair in the early months postinjury. ${ }^{26}$

\section{Clinical indicators of recovery}

The clinical examination is relatively insensitive to early features of nerve regeneration. Early muscle reinnervation may not generate sufficient force for muscle movement. Secondary changes in a limb, such as joint contractures, may interfere with muscle movement despite successful reinnervation. A mobile Tinel's phenomenon (tingling in the distribution of a peripheral nerve with percussion) may be able to detect advancing fine fibre 
regeneration following PNT; this, however, may not necessarily imply that functional recovery will follow. ${ }^{27-29}$

\section{Electrodiagnostic monitoring}

Serial EMG assessments are more sensitive than the clinical examination to detect nerve regeneration. Early reinnervated, or 'nascent' MUs may be detectable on EMG when there is no clinical improvement (figure 1). However, though encouraging, the detection of nascent MUs (and reduction in the density of fibrillation potentials) does not guarantee subsequent functional recovery. The major limitation of EMG in monitoring for signs of nerve regeneration is that EMG will not show any evidence of nerve recovery until a fairly large number of mature axons have reached the muscles being studied, despite the fact that axonal regeneration may have been advancing proximal to this point. This limitation makes decisions regarding the potential success of spontaneous nerve regeneration more difficult and may prolong the period of observation to the detriment of those patients who actually do require surgical nerve repair.

\section{Intraoperative electrophysiological assessments}

Intraoperative electrophysiological studies are a major advance in management of PNT, and were developed over four decades ago. Studies are performed following surgical exposure of the nerve and involve the generation of compound nerve action potentials (CNAPs) by stimulating and recording electrodes applied directly to the nerve trunk (figure 4). ${ }^{30}$ Intraoperative electrophysiological studies have the distinct advantage of permitting directed and detailed study of an injured nerve trunk, thus enabling thorough assessment of lesions in continuity that may be inaccessible to standard NCS. In the acute setting, they may suggest the presence or absence of intact axons running through the lesion. To evaluate for evidence of nerve regeneration, intraoperative assessments must be delayed for 2-3 months to allow time for useful numbers of axons, over $5 \mu$ in diameter and with early myelination, to have penetrated the lesion and reach the nerve segment just distal to it before a CNAP can be recorded. ${ }^{32}$ Technical and equipment factors need to be controlled and considered in the interpretation of intraoperative studies. A further drawback of intraoperative assessments of nerve regeneration is that an invasive procedure is necessary and the development of non-invasive techniques to detect nerve regeneration before muscle reinnervation will represent an additional critical advance in the management of nerve injury.

\section{Visualising nerve regeneration}

\section{Diffusion tensor tractography}

Ultrasound or standard MRN are unable to fully discriminate between neurotmesis and axonotmesis, particularly when the nerve remains in continuity. Diffusion tensor tractography (DTT) represents a recent development in MRI that may revolutionise this aspect of the diagnosis and monitoring of PNT (figures 1 and 3).

Diffusion weighted imaging takes advantage of anisotropic (or directional) movement of water molecules in imaged tissues. Peripheral nerves are comprised of longitudinal axonal tracts resulting in relatively free diffusion of water molecules in the direction of the tract, but restricted movement perpendicular to the nerve. ${ }^{33}$ Diffusion tensor imaging (DTI) has been extensively applied to the brain and can demonstrate integrity of nerve tracts through measurement of fractional anisotropy (FA) and related indices. Limitations of peripheral nerve DTI include spatial and contrast resolution and relatively low signal-to-noise ratio, but if these issues can be overcome peripheral nerve DTI opens opportunities to non-invasively evaluate nerve integrity.

There have been promising applications of DTT to PNT and regeneration. In healthy participants, bundles of fibres may be tracked through a normal nerve. ${ }^{34}$ Following axonal injury in animal models, trackable fibres terminate at the point of nerve injury within hours of nerve trauma. ${ }^{35}{ }^{36}$ With nerve regeneration, fibres may be seen to extend beyond the point of nerve injury, restoring a relatively normal tract through regeneration with a corresponding increase in FA values at the point of nerve injury. ${ }^{35}$ In humans, nerve regeneration may be visualised using DTT, correlating with evidence of recovery on clinical and electrodiagnostic testing. ${ }^{37} 38$ Peripheral nerve DTT has also been applied to delineate normal nerve fascicles in relation to peripheral nerve sheath tumours to allow planning of surgical approaches. ${ }^{39}$ Although further investigation of this emerging technique is needed, DTT has the potential to enable early detection of peripheral nerve regeneration and thus, facilitate earlier determination of those patients who may or may not benefit from surgical repair of nerve injury. DTT may also serve as a useful technique to monitor axonal regeneration in the period during which EMG studies are of limited value, specifically early after nerve injury. ${ }^{37}$

The question remains, however, whether regenerating axons detected by DTT in human participants will be large enough, in required numbers and with enough myelination to accurately predict useful recovery in the distribution of the nerve being studied; recovery of DTI parameters in an animal model of nerve injury correlated with return of larger calibre axons. ${ }^{35}$ Separately, current reports evaluating DTT monitoring of regeneration have involved patients with nerve transections; therefore, further data regarding the detection of regenerating axons in lesions in continuity are needed.

It is predicted that imaging advances will herald an era of clinician-directed, multimodality assessment of nerve injury, which may provide greatly enhanced non-invasive functional and anatomic assessment of nerve injury, estimations of prognosis and determination of optimum treatment modalities. ${ }^{40}$

\section{SURGICAL ADVANCES}

Standard approaches to the management of PNT include avoidance of further traumatic insults, physical therapy and splinting, and surgical exploration (in many cases with operative CNAP recordings) and neurorrhaphy of severely damaged nerves. ${ }^{41}$ While these standard approaches may be satisfactory for many nerve injuries, progressive surgical techniques and medical management may improve functional outcomes. The following sections highlight areas in which promising developments have been reported.

\section{Surgical repair}

In many instances, PNT will require operative exploration. The choice of technique and timing of surgery depends on the mechanism of injury, the length of the damaged nerve segment and the extent of surrounding tissue damage. ${ }^{25}$ While the details of specific surgical approaches fall outside the scope of this review (and readers are directed to specific reviews on the topic $^{42}$ ), surgical and biotechnological advances have contributed to improved treatment outcomes for peripheral nerve surgery. In general, nerves may be repaired by reconnecting proximal and distal stumps (neurorrhaphy), or by joining proximal and distal stumps by means of a nerve graft or conduit to bridge a nerve defect. Procedures connecting uninjured nerves to the distal portion of an injured nerve (neurotisation) are 
Figure 4 Illustration of intraoperative compound nerve action potential (CNAP) studies of a nerve injury in continuity. A bipolar or tripolar (pictured) stimulating electrode is placed proximal to the injured segment and the recording electrode placed distal to the injured segment (A). In normal nerves a high amplitude CNAP can be recorded (B). If intraoperative CNAP recordings are performed shortly after injury, the presence of residual intact axons may be detected (C). CNAP studies performed after a delay of 2-3 months may be useful to detect evidence of axon regeneration across an injured nerve segment (D).
Stimulating

A

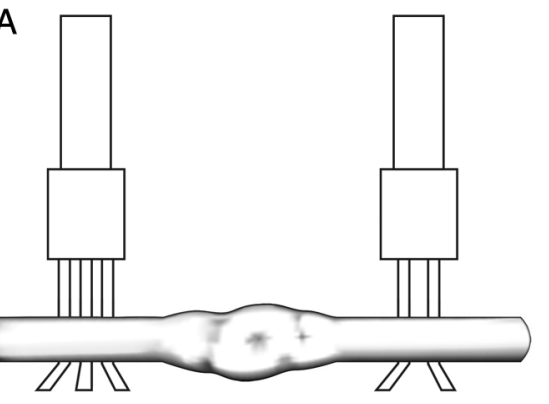

Injured nerve segment

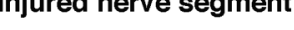

\section{Normal CNAP}

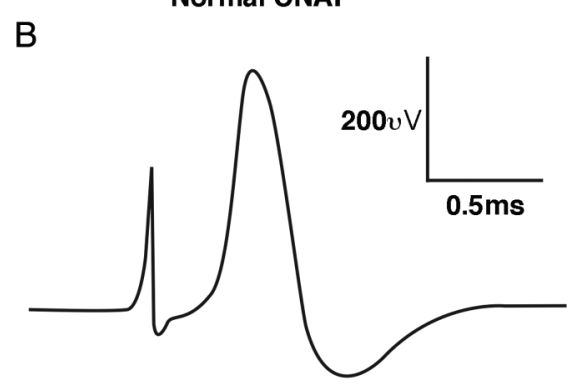

C Shortly after injury

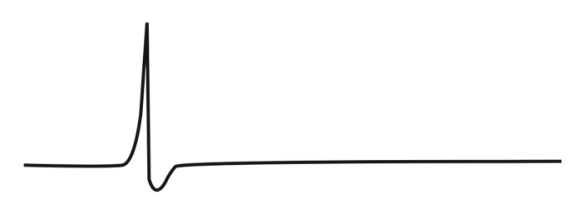

No or few intact axons

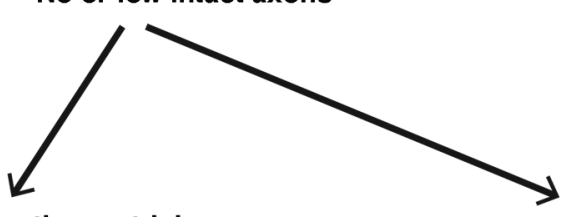

2-3 months post-injury

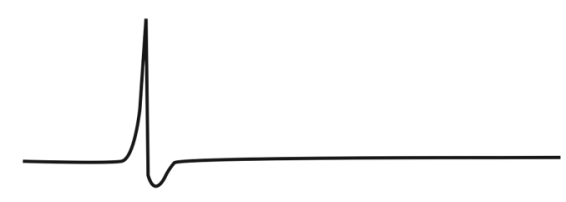

No recovery

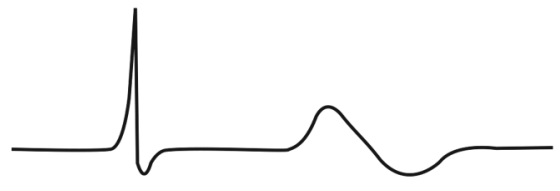

Regenerating axons sometimes employed if direct repair of the injured nerve is not possible.

\section{Management of nerve root avulsion}

Severe brachial plexus trauma may be complicated by nerve root avulsion, which makes assessment and management of the nerve injury more difficult. Specifically, patients with nerve root avulsion will not demonstrate spontaneous improvement and require specialised approaches to brachial plexus reconstruction. Relatively rapid loss of anterior horn cells follows motor axotomy, perhaps underpinning the importance of early intervention in these cases. Nerve avulsion may be apparent clinically, for example, patients with dorsal nerve root avulsion may develop severe neuropathic pain within days of the injury. Brachial plexus imaging provides the most valuable information and is indicated in the work-up of all patients presenting with brachial plexus trauma. Nerve root avulsion may be apparent on MRI or ultrasound studies, ${ }^{43-45}$ although CT or digitalsubtraction myelography may be required in some instances. A pseudomeningocoele may be seen in some cases.

Reimplantation of the avulsed motor root provides the opportunity for anterior horn cells to extend axons into the root and improve the chances of functional recovery. ${ }^{46}$ This is particularly relevant for proximal muscle groups, but functional hand muscle recovery is usually disappointing. This technique is being performed selectively in a few centres. Surgical repair of avulsed ventral roots by reimplantation is only usually successful within a month of the injury ${ }^{47}$ and this provides an argument for ultraearly surgical intervention following brachial plexus trauma. ${ }^{48}$ Clinical outcomes are improved when surgery is performed within 7 days of injury and the likelihood of recovery diminishes with increasing delays. ${ }^{49}$ In addition, surgery is technically easier very early after injury, as there has been less time for tissue fibrosis to occur and recoiling distal stumps of ruptured nerves may be more easily retrieved. The drawback of ultraearly surgery is that intraoperative CNAP recordings are rendered less informative for the evaluation of non-avulsed nerve trunks as sufficient time has not elapsed for any potential reinnervation to occur.

The more common alternative approach is a nerve transfer, where a healthy donor nerve is coapted to the distal stump or the continuation of the avulsed root $^{50}$ In some instances, this may include transposition of the contralateral C7 nerve root; however, concerns about donor side morbidity have limited the 
use of this approach. ${ }^{51}$ Different nerve transfers (see Neurotisation section below) can be considered to target recipients with available, expendable donors.

Brachial plexus trauma with nerve root avulsion may result in severe and intractable pain, resistant to medical treatment. Surgical reimplantation of nerve roots alone may improve pain, irrespective of functional outcomes, and additional nerve reconstruction for proximal brachial plexus injuries may also be useful to reduce pain even when functional recovery is poor. In patients with refractory pain, surgical lesions of the dorsal root entry zone may represent a viable treatment approach. This technique is particularly useful at improving lancinating paroxysmal pain, but is less effective at managing persistent background burning pain. ${ }^{52}$ Electrical motor cortex stimulation has shown some benefit in reducing this background pain $^{53}$ and combination approaches may prove to be most worthwhile.

\section{Refinement of nerve grafting techniques}

When nerve trauma results in a gap between the viable nerve stumps and when primary neurorrhaphy is not possible, a nerve graft or conduit may need to be interposed in order to provide a pathway for regenerating axons. Failure to provide this regeneration pathway usually results in the interspersion of scar tissue and failed regeneration.

Autologous nerve grafts are the optimum material for bridging defects in injured nerves, as these contain all the necessary microstructural elements to facilitate axonal migration without antigenic components that may occur with artificial or cadaveric grafts. However, donor nerves (typically sural, superficial radial and/or medial antebrachial cutaneous nerves) occasionally may not provide sufficient tissue to satisfactorily bridge a long nerve defect or a defect in a large calibre nerve.

Synthetic conduits have been promoted as an alternate approach to nerve autografts, but axonal growth through these hollow polymer tubes may be limited in defects of more than $10 \mathrm{~mm},{ }^{54}$ probably because they lack the microarchitecture of the native nerve. ${ }^{55}$ In general, conduits are used for injuries to minor nerves, for example, digital or other small important sensory nerves, and are avoided for major nerve injuries. Synthetic scaffolds have been developed with cellular guidance channels that facilitate propagation of Schwann cell processes, which may improve the chances of successful nerve regeneration. $^{55-57}$

Nerve tissue allografts from cadaveric donors provide necessary cellular structures, but require immunosuppression for up to 18 months to prevent rejection ${ }^{58} 59$ and the complications of immunosuppression are seldom justified. Processed, acellular nerve allografts eliminate the need for immunosuppression, but inflammatory reactions may still rarely result in scarring that may impede nerve regeneration. ${ }^{60}$ Functional outcomes are encouraging, with a recent study demonstrating improvement in $87 \%$ of patients with allografts for gaps of between 5 and $50 \mathrm{~mm}^{61}$

Finally, animal-derived, acellular, extracellular matrix nerve cuffs, for example, using porcine small intestinal submucosa, provide the necessary scaffold to guide axonal regeneration, without issues of immunogenicity. ${ }^{62}$

Neurotrophic factors, such as neurotrophins, insulin-like growth factors, and glial cell line-derived neurotrophic factors, secreted by neuronal and non-neuronal cells in the proximal and distal nerve stump drive the process of nerve regeneration in vivo. ${ }^{63}$ The absence of these growth factors in synthetic conduits may contribute to failure of axonal regeneration over longer graft lengths. Adding one or more of these neurotrophic growth factors improves axonal regeneration. ${ }^{64} 65$ Dosing and drug kinetics play an important role in the efficacy of growth factor strategies and excessive delivery can actually impede axonal regeneration. ${ }^{66} \mathrm{~A}$ number of biotechnology strategies are being used to develop optimum delivery methods, ${ }^{67}$ but presently none are approved for use in clinical practice.

\section{Neurotisation procedures}

When there is extensive proximal nerve injury or nerve root avulsion, nerve grafting strategies typically fail. Neurotisation procedures, involving the suturing of an uninjured nerve to the distal portion of an injured nerve, provide an alternative route for axonal regeneration enabling reinnervation of the target muscles. ${ }^{68}$ Neurotisation bypasses the zone of nerve injury and brings the regenerating axons closer to the target muscle, thus improving the chances of recovery. A number of techniques have been developed, and muscle reinnervation and clinical improvement has been reported with anastomosis of an intact nerve to an injured nerve in an end-to-end, end-to-side and even side-to-side fashion. ${ }^{69}$ Neurotisation involves transferring a functioning, expendable nerve, branch or fascicle to a more important, non-functioning recipient. Examples would include the use of intercostal nerves, the distal spinal accessory nerve, contralateral C7, a triceps branch, or a fascicle of the ulnar or median nerve. Direct muscle neurotisation may be an option for those patients in whom there is no distal nerve stump remaining. $^{70}$

When reconstructive surgery must be substantially delayed, there is a concern that the connective tissue elements (such as Schwann cells) in the distal nerve stump are less able to receive regenerating axons if they have remained denervated for a prolonged period. 'Sensory nerve protection' has been proposed as a means of providing trophic support to the distal stump of a motor nerve by coapting a healthy sensory nerve while awaiting formal repair procedures. ${ }^{71}$ However, there are few situations in which this approach is favoured over reconstruction combining grafting and nerve transfers, which generally provide the best results. $^{72}$

\section{Other reconstructive procedures}

In patients with severe functional deficits, in whom attempts at nerve repair are unsuccessful or impossible, other reconstructive procedures may be used. These may include tendon transfer, free functioning muscle transfer and bony procedures, including fusion (arthrodesis). There are many procedures described and choice of approach depends on the extent of nerve and soft tissue injury, as well as the functional goals. Extensive rehabilitation and retraining is necessary to optimise the outcome of these reconstructive procedures.

\section{Nanotechnology and axon splicing}

One major limitation of current surgical approaches is that they inevitably involve Wallerian degeneration and subsequent axonal regeneration through residual connective tissue channels, and rely on a complex array of cellular and biochemical processes. Successful muscle reinnervation must occur within several years from the date of injury (commonly stated as 2 years although longer periods have been suggested), or irreversible Schwann cell deterioration and muscle fibrosis and fatty infiltration may occur. $^{7374}$ Thus, the time taken for regeneration of axons from a proximal limb nerve injury to a distal muscle (eg, from the brachial plexus to the intrinsic hand muscles) may exceed this time frame. In addition, the number and accuracy of axons reaching their target muscle is markedly subphysiological and as such, 
muscle reinnervation is inevitably incomplete. ${ }^{63}$ Prevention of Wallerian degeneration is an optimal, perhaps ambitious, approach.

Nanotechnology developments may render this approach feasible in the future. Microcutting tools are able to make neat and precise cuts to individual axons, while axon ends can be manipulated using dielectrophoretic force, and axon stumps fused by electrofusion. ${ }^{75-78}$ Given that many individual axon repairs will be necessary to achieve a functional reconnection, these processes will need to be automated, and clearly such a procedure must be performed very soon after nerve injury to avoid the cascade of distal axonal degeneration. Robot-assisted microsurgical approaches have emerged as a promising tool ${ }^{79}$ and this technology may be harnessed in such a way as to make individual axon repair feasible in the future.

\section{REHABILITATION AND NON-SURGICAL APPROACHES}

Rehabilitation strategies are vitally important following PNT to minimise permanent physical deficits and to promote functional adaptation to compensate for these. Range of motion, stretching exercises and biofeedback are useful to prevent contractures and secondary deformities. Sensory re-education techniques have been developed to improve sensory function after reinnervation and to maintain cortical sensory organisation, ${ }^{80}$ though evidence of the efficacy is presently limited. ${ }^{81}$ Novel approaches may enhance the possibility of nerve regeneration and functional recovery.

\section{Repetitive nerve stimulation and activity-dependent treatments}

Both PNT and the resultant relative inactivity of the muscle result in muscle atrophy and alteration in the biochemical characteristics of muscle fibres, with these changes becoming irreversible with time. ${ }^{82}$ However, there have been reports of progressive functional recovery occurring even 5 years after the initial injury following nerve repair, ${ }^{6}$ suggesting that residual satellite cells may retain the capacity for muscle regeneration even after a more prolonged period of denervation. ${ }^{83}$

In addition to muscle factors, Schwann cell regenerative capacity is time limited, such that delay of effective anastomosis of injured nerve stumps and hence, prolonged denervation of distal Schwann cells results in a reduced proportion of axons that regenerate relative to a nerve that has been promptly reanastomosed. ${ }^{84}{ }^{85}$ Besides any iatrogenic delay, the process of initiation of axonal regeneration at the anastomosis site may be delayed and a period of up to 3-4 weeks may be required for all axons to begin regenerating across the anastomosis, ${ }^{86}$ further challenging the timeliness of repair mechanisms. Reduced regenerative potential through attenuated regeneration apparatus and muscle atrophy impacts on the ability of reinnervated muscle to regain normal morphology and function, particularly when long time intervals are expected for regeneration from proximal injuries.

To overcome the issue of muscle and Schwann cell degradation, repetitive nerve stimulation protocols have been devised. In animal studies, low-intensity electrical stimulation of a repaired nerve for $1 \mathrm{~h}$ resulted in increased axonal regeneration, relative to animals receiving sham stimulation. Increased efficiency of axonal outgrowth through the surgical site was proposed as a mechanism for this improvement and the accuracy of target reinnervation was improved in stimulated nerves. ${ }^{87} 88$ Similarly, in patients undergoing carpal tunnel release surgery for severe carpal tunnel syndrome, MU number estimation studies identified a significant increase in MU numbers in patients who underwent $1 \mathrm{~h}$ of nerve stimulation following the release procedure. ${ }^{89}$ Exercise is also effective at improving muscle reinnervation, and reducing neuropathic pain and mechanical allodynia, ${ }^{90}$ but may be less effective than nerve stimulation at promoting axon regeneration.

\section{Prevention and management of traumatic neuroma}

Development of a traumatic neuroma following nerve injury or its treatment may introduce a significant source of neuropathic pain, which may compound the disability associated with the neurological impairment. Any traumatic nerve injury resulting in axonal disruption, including surgical trauma, may result in the development of neuroma. This becomes more challenging, as surgical resection of a painful neuroma may result in recurrence at the new cut end, initiating an additional cycle of injury and aberrant repair. A number of surgical techniques have been proposed for minimising neuroma formation following surgical nerve resection; ${ }^{92}$ targeted muscle reinnervation is a particularly promising method to minimise development of painful neuroma postamputation. ${ }^{93}$ Management of neuroma developing after incomplete nerve trauma is less developed and current strategies include adjuvant neuropathic pain agents, local anaesthetic and/or corticosteroid infiltration, and nerve ablative therapies, such as phenol injection.

Besides promoting axonal regeneration, repetitive nerve stimulation may also be a useful treatment modality in managing painful traumatic neuromas following nerve injury. Peripheral or spinal repetitive electrical stimulation may reduce pain associated with postamputation neuromas. ${ }^{94}$ Low-frequency transcutaneous magnetic stimulation reduced neuroma-associated pain in one small preliminary study. ${ }^{95}$ These non-invasive nerve stimulation techniques may be useful adjunctive treatments following PNT or may have a role when conventional approaches have failed.

\section{Central adaptation after nerve injury}

Despite successful reinnervation of target muscles, functional recovery from PNI may be relatively poor. Much of this functional impairment can be apportioned to reduced axon numbers and altered MU morphology. ${ }^{96}$ In addition, regenerating axons may reach target tissues with poor specificity such that the original function of the axon may not be matched with the function of the target organ. ${ }^{97}$ This phenomenon may be more pronounced when greater distances regeneration are involved.

Central adaptation may play an important role in recovery, in particular to overcome the inaccuracy of nerve regeneration, although it remains more limited to disturbed sensory localisation or fine motor control. ${ }^{98}$ Central maladaptation may be an important factor underlying the development of neuropathic pain, dysaesthesia and dystonia following PNI and repair. Harnessing and augmenting these central processes may be a future consideration in planning treatment and rehabilitation strategies.

Perhaps the most notable example of the influence of central adaptation after nerve injuries may be seen in obstetric brachial plexus palsy. Many children with this injury recover well with conservative or operative approaches; however, up to $15 \%$ have persistent severe deficits. A subgroup of these patients has demonstrable MUs on EMG, but persistently impaired motor skills using these reinnervated muscles. ${ }^{99}$ Experimental studies have suggested that this may be due to a developmental apraxia, with defective motor programming early in infancy. ${ }^{100} 101$

Central adaptation processes occur both at the spinal, cortical and subcortical levels. Animal models have suggested spinal 
remodelling contributes to recovery following nerve injury, with compensatory changes in spinal architecture seen after segmental axonal loss. ${ }^{102}$ Changes at the spinal level include altered neuronal and reflex excitability, reduced inhibitory interneuronal inputs, and remodelling of spinal circuitry and somatotopic maps. $^{98}$

Studies of the somatosensory and motor cortices are most frequently used to describe brain changes following PNT. Rapid changes in somatosensory and motor cortical maps occur after injury. ${ }^{103} 104$ The areas representing the denervated territories become inactive, but adjacent areas expand to occupy parts of that territory. ${ }^{105}$ With reinnervation, the cortical maps revert closer to normal, ${ }^{106} 107$ but cortical representations remain distorted.

\section{Controlling central remodelling}

A key question is how to effectively guide central reorganisation while avoiding the maladaptive outcomes described above. A number of strategies have been developed and further investigation is warranted.

Vigorous rehabilitation plays an important role in the recovery from PNT by facilitating functional adaptation and preventing secondary tissue effects, such as contractures. In addition, manipulating the flow of sensory and motor inputs can manage maladaptive cortical plasticity. As an example, sensory discrimination or motor tasks result in the expansion of appropriate cortical maps and refinement of receptive fields. ${ }^{108}$

More active interventions may also help shape central processes. Repetitive nerve stimulation results in brain cortical changes that outlast the duration of the stimulus. ${ }^{109}$ Synchronised peripheral nerve stimulation paired with transcranial magnetic stimulation of the primary motor cortex produces lasting increases in excitability of targeted muscles. ${ }^{110}$ Isolated repetitive brain stimulation has been shown to be effective in reducing chronic pain; ${ }^{111}$ this, however, remains untested in other functional deficits following peripheral nerve injury. These approaches may provide a method to more actively manage severe PNT and further investigation may identify novel treatment strategies.

\section{CONCLUSION}

The peripheral nervous system is unique in its ability to regenerate even after severe injuries, and this process involves a complex interplay of cellular and anatomic factors. The approach of 'expectant observation' may produce suboptimal functional outcomes or result in excessive delay until definitive management is instituted. Intraoperative electrophysiological studies may provide the necessary information to enable timely decision-making, but to date these are necessarily invasive.

On the basis of novel diagnostic techniques, in particular the advances in peripheral nerve imaging, patients may be better triaged to receive prompt surgical intervention where needed and avoid invasive procedures when spontaneous regeneration is likely. We also suggest that further exploration of non-invasive strategies to augment nerve regeneration processes, such as modulation of central and axonal plasticity through repetitive stimulation and functional retraining paradigms, may provide further benefit for patients with moderate and severe nerve injury, including those patients in whom surgical intervention is not needed.

Acknowledgements NGS gratefully acknowledges funding from the National Health and Medical Research Council and the Motor Neurone Disease Research Institute of Australia (grant \#1039520).
Contributors NGS was involved in drafting the manuscript, preparation of figures, revising the manuscript and approval of the final manuscript. RJS, DGK and MK were involved in revising the manuscript and approval of the final manuscript.

Funding Motor Neurone Disease Research Institute of Australia. National Health and Medical Research Council.

Competing interests None declared.

Provenance and peer review Commissioned; externally peer reviewed.

\section{REFERENCES}

1 Kurtzke JF. The current neurologic burden of illness and injury in the United States. Neurology 1982;32:1207-14.

2 Kaiser R, Waldauf $P$, Haninec $P$. Types and severity of operated supraclavicular brachial plexus injuries caused by traffic accidents. Acta Neurochir 2012;154:1293-7.

3 Dubuisson A, Kline DG. Indications for peripheral nerve and brachial plexus surgery. Neurol Clin 1992;10:935-51.

4 Dubuisson AS, Kline DG. Brachial plexus injury: a survey of 100 consecutive cases from a single service. Neurosurgery 2002;51:673-82; discussion 682-3.

5 Murovic JA. Upper-extremity peripheral nerve injuries: a Louisiana State University Health Sciences Center literature review with comparison of the operative outcomes of 1837 Louisiana State University Health Sciences Center median, radial, and ulnar nerve lesions. Neurosurgery 2009;65(4 Suppl):A11-17.

$6 \mathrm{Kim}$ DH, Cho YJ, Tiel RL, et al. Outcomes of surgery in 1019 brachial plexus lesions treated at Louisiana State University Health Sciences Center. J Neurosurg 2003;98:1005-16.

7 Birch R. Surgical disorders of the peripheral nerve. 2nd edn. London: Springer-Verlag, 2011.

8 Seddon HJ. Peripheral nerve injuries, Medical Research Council Special Report Series No. 282. London: Her Majesty's Stationery Office, 1954.

9 Sunderland S. A classification of peripheral nerve injuries producing loss of function. Brain 1951;74:491-516.

10 Erra C, Granata G, Liotta G, et al. Ultrasound diagnosis of bony nerve entrapment: case series and literature review. Muscle Nerve 2013;48:445-50.

11 Spinner RJ, Atkinson JL, Scheithauer BW, et al. Peroneal intraneural ganglia: the importance of the articular branch. Clinical series. J Neurosurg 2003;99:319-29.

12 Visser LH. High-resolution sonography of the common peroneal nerve: detection of intraneural ganglia. Neurology 2006;67:1473-5.

13 Padua L, Di Pasquale A, Liotta G, et al. Ultrasound as a useful tool in the diagnosis and management of traumatic nerve lesions. Clin Neurophysiol 2013;124:1237-43.

14 Abell MR, Hart WR, Olson JR. Tumors of the peripheral nervous system. Hum Pathol 1970;1:503-51.

15 Hobson-Webb LD, Walker FO. Traumatic neuroma diagnosed by ultrasonography. Arch Neurol 2004;61:1322-3.

16 West GA, Haynor DR, Goodkin R, et al. Magnetic resonance imaging signal changes in denervated muscles after peripheral nerve injury. Neurosurgery 1994;35:1077-85.

17 Simon NG, Ralph JW, Lomen-Hoerth C, et al. Quantitative ultrasound of denervated hand muscles. Muscle Nerve 2014. Nov 11. doi: 10.1002/ mus.24519.

18 Koltzenburg M, Bendszus M. Imaging of peripheral nerve lesions. Curr Opin Neurol 2004;17:621-6

19 Simon NG, Ralph JW, Chin C, et al. Sonographic diagnosis of true neurogenic thoracic outlet syndrome. Neurology 2013;81:1965.

20 Simon NG, Ralph JW, Poncelet AN, et al. A comparison of ultrasonographic and electrophysiologic 'inching' in ulnar neuropathy at the elbow. Clin Neurophysiol 2015;126:391-8.

21 Gallardo E, Noto YI, Simon NG. Ultrasound in the diagnosis of peripheral neuropathy: structure meets function in the neuromuscular clinic. J Neurol Neurosurg Psychiatry 2015;86:1066-74.

22 Padua L, Aprile I, Pazzaglia C, et al. Contribution of ultrasound in a neurophysiological lab in diagnosing nerve impairment: a one-year systematic assessment. Clin Neurophysiol 2007;118:1410-16.

23 Koenig RW, Schmidt TE, Heinen CP, et al. Intraoperative high-resolution ultrasound: a new technique in the management of peripheral nerve disorders. J Neurosurg 2011;114:514-21.

24 Boon AJ, Bailey PW, Smith J, et al. Utility of ultrasound-guided surface electrode placement in lateral femoral cutaneous nerve conduction studies. Muscle Nerve 2011;44:525-30.

25 Spinner RJ, Kline DG. Surgery for peripheral nerve and brachial plexus injuries or other nerve lesions. Muscle Nerve 2000;23:680-95.

26 Kline DG. Timing for brachial plexus injury: a personal experience. Neurosurg Clin N Am 2009;20:24-6.

27 Tinel J. Le signe du fourmillement dans les lésions des nerfs périphériques. Presse Méd 1915;47:388-9. 
28 Napier JR. The significance of Tinel's sign in peripheral nerve injuries. Brain 1949;72:63-82.

29 Henderson WR. Clinical assessment of peripheral nerve injuries Tinel's test. Lancet 1948:252:801-5

30 Crum BA, Strommen JA. Peripheral nerve stimulation and monitoring during operative procedures. Muscle Nerve 2007;35:159-70.

31 Robert EG, Happel LT, Kline DG. Intraoperative nerve action potential recordings: technical considerations, problems, and pitfalls. Neurosurgery 2009;65(4 Suppl): A97-104

32 Kline DG, Hackett ER. Reappraisal of timing for exploration of civilian peripheral nerve injuries. Surgery 1975;78:54-65.

33 Simon NG, Kliot M. Diffusion weighted MRI and tractography for evaluating peripheral nerve degeneration and regeneration. Neural Regen Res 2014;9:2122-4

34 Hiltunen J, Suortti T, Arvela $\mathrm{S}$, et al. Diffusion tensor imaging and tractography of distal peripheral nerves at 3T. Clin Neurophysiol 2005;116:2315-23.

35 Takagi T, Nakamura M, Yamada M, et al. Visualization of peripheral nerve degeneration and regeneration: monitoring with diffusion tensor tractography. Neuroimage 2009;44:884-92.

36 Lehmann HC, Zhang J, Mori S, et al. Diffusion tensor imaging to assess axonal regeneration in peripheral nerves. Exp Neurol 2010;223:238-44.

37 Simon NG, Narvid J, Cage T, et al. Visualizing axon regeneration after peripheral nerve injury with magnetic resonance tractography. Neurology 2014;83:1382-4.

38 Meek MF, Stenekes MW, Hoogduin HM, et al. In vivo three-dimensional reconstruction of human median nerves by diffusion tensor imaging. Exp Neurol 2006;198:479-82.

39 Simon NG, Cage T, Narvid J, et al. High-resolution ultrasonography and diffusion tensor tractography map normal nerve fascicles in relation to schwannoma tissue prior to resection. J Neurosurg 2014;120:1113-17.

40 Selmonosky CA. Sonographic diagnosis of true neurogenic thoracic outlet syndrome. Neurology 2014;83:198.

41 Simon NG, Kiernan MC. Mononeuropathy. In: Hilton-Jones D, Turner MR, eds. Oxford textbook of neuromuscular disorders. Oxford: Oxford University Press, 2014:93-114

42 Kline DG. Nerve surgery as it is now and as it may be. Neurosurgery 2000;46:1285-93.

43 Zhu YS, Mu NN, Zheng MJ, et al. High-resolution ultrasonography for the diagnosis of brachial plexus root lesions. Ultrasound Med Biol 2014:40:1420-6.

44 Doi K, Otsuka K, Okamoto Y, et al. Cervical nerve root avulsion in brachial plexus injuries: magnetic resonance imaging classification and comparison with myelography and computerized tomography myelography. J Neurosurg 2002;96(3 Suppl):277-84.

45 Kline DG, Hudson AR. Diagnosis of root avulsions. J Neurosurg 1997;87:483-4

46 Havton LA, Carlstedt T. Repair and rehabilitation of plexus and root avulsions in animal models and patients. Curr Opin Neurol 2009:22:570-4.

47 Fournier HD, Mercier P, Menei P. Repair of avulsed ventral nerve roots by direct ventral intraspinal implantation after brachial plexus injury. Hand Clin 2005;21:109-18.

48 Birch R. Timing of surgical reconstruction for closed traumatic injury to the supraclavicular brachial plexus. J Hand Surg 2014. Jul 8. pii: 1753193414539865

49 Birch R. The closed supraclavicular lesion. In: Birch R, ed. Surgical disorders of the peripheral nerves. 2nd edn. London: Springer, 2011:375-428.

50 Addas BM, Midha R. Nerve transfers for severe nerve injury. Neurosurg Clin N Am 2009;20:27-38

51 Sammer DM, Kircher MF, Bishop AT, et al. Hemi-contralateral C7 transfer in traumatic brachial plexus injuries: outcomes and complications. J Bone Joint Surg 2012;94:131-7.

52 Aichaoui $F$, Mertens $P$, Sindou M. Dorsal root entry zone lesioning for pain after brachial plexus avulsion: results with special emphasis on differential effects on the paroxysmal versus the continuous components. A prospective study in a 29-patient consecutive series. Pain 2011;152:1923-30.

53 Ali M, Saitoh Y, Oshino S, et al. Differential efficacy of electric motor cortex stimulation and lesioning of the dorsal root entry zone for continuous vs paroxysmal pain after brachial plexus avulsion. Neurosurgery 2011;68:1252-7.

54 Daly WT, Knight AM, Wang $H$, et al. Comparison and characterization of multiple biomaterial conduits for peripheral nerve repair. Biomaterials 2013;34:8630-9.

55 Ribeiro-Resende VT, Koenig B, Nichterwitz $S$, et al. Strategies for inducing the formation of bands of Bungner in peripheral nerve regeneration. Biomaterials 2009:30:5251-9.

56 Cunha C, Panseri S, Antonini S. Emerging nanotechnology approaches in tissue engineering for peripheral nerve regeneration. Nanomedicine 2011;7:50-9.

57 Bozkurt A, Deumens R, Beckmann C, et al. In vitro cell alignment obtained with a Schwann cell enriched microstructured nerve guide with longitudinal guidance channels. Biomaterials 2009:30:169-79.

58 Grand AC, Myckatyn TM, Mackinnon SE, et al. Axonal regeneration after cold preservation of nerve allografts and immunosuppression with tacrolimus in mice. J Neurosurg 2002;96:924-32.
59 Mackinnon SE, Doolabh VB, Novak CB, et al. Clinical outcome following nerve allograft transplantation. Plast Reconstr Surg 2001;107:1419-29.

60 Kehoe S, Zhang XF, Boyd D. FDA approved guidance conduits and wraps for peripheral nerve injury: a review of materials and efficacy. Injury 2012:43:553-72.

61 Brooks DN, Weber RV, Chao JD, et al. Processed nerve allografts for peripheral nerve reconstruction: a multicenter study of utilization and outcomes in sensory, mixed, and motor nerve reconstructions. Microsurgery 2012;32:1-14.

62 Smith RM, Wiedl C, Chubb P, et al. Role of small intestinal submucosa (SIS) as a nerve conduit: preliminary report. J Invest Surg 2004;17:339-44.

63 Fu SY, Gordon T. The cellular and molecular basis of peripheral nerve regeneration. Mol Neurobiol 1997;14:67-116.

64 Cao X, Shoichet MS. Investigating the synergistic effect of combined neurotrophic factor combination gradients to guide axonal growth. Neuroscience 2003;122:381-9.

65 Madduri S, Feldman K, Tervoort T, et al. Collagen nerve conduits releasing the neurotrophic factors GDNF and NGF. J Control Release 2010;143:168-74.

66 Conti AM, Brimijoin S, Miller LJ, et al. Suppression of neurite outgrowth by high-dose nerve growth factor is independent of functional p75NTR receptors. Neurobiol Dis 2004;15:106-14.

67 Ramburrun $\mathrm{P}$, Kumar $\mathrm{P}$, Choonara YE, et al. A review of bioactive release from nerve conduits as a neurotherapeutic strategy for neuronal growth in peripheral nerve injury. Biomed Res Int 2014;2014:132350.

68 Songcharoen P, Wongtrakul S, Spinner RJ. Brachial plexus injuries in the adult. nerve transfers: the Siriraj Hospital experience. Hand Clin 2005;21:83-9.

69 Cage TA, Simon NG, Bourque $S$, et al. Dual reinnervation of biceps muscle after side-to-side anastomosis of an intact median nerve and a damaged musculocutaneous nerve. J Neurosurg 2013;119:929-33.

70 Brunelli GA, Brunelli GR. Direct muscle neurotization. J Reconstr Microsurg 1993:9:81-90

71 Bain JR, Veltri KL, Chamberlain $\mathrm{D}$, et al. Improved functional recovery of denervated skeletal muscle after temporary sensory nerve innervation. Neuroscience 2001;103:503-10.

72 Kline DG, Tiel RL. Direct plexus repair by grafts supplemented by nerve transfers. Hand Clin 2005;21:55-69.

73 Kline DG, Hudson AR. Surgical repair of acute peripheral nerve injuries: timing and technique. In: Morley T. ed. Controversies in neurosurgery. Philadelphia: W. B. Saunders Co., 1976:184-97.

74 Sunderland S. Nerve and nerve injuries. Edinburgh: Livingstone, 1978.

75 Chang W, Sretevan D, Kliot M. A tribute to Dr. David Kline: a new approach to an old peripheral nerve problem-splicing instead of regenerating disrupted axons. Neurosurgery 2009;65(4 Suppl):A52-4.

76 Chang WC, Hawkes EA, Kliot $M$, et al. In vivo use of a nanoknife for axon microsurgery. Neurosurgery 2007;61:683-91.

77 Chang WC, Kliot M, Sretavan DW. Microtechnology and nanotechnology in nerve repair. Neurol Res 2008;30:1053-62.

78 Sretavan DW, Chang W, Hawkes $\mathrm{E}$, et al. Microscale surgery on single axons. Neurosurgery 2005;57:635-46.

79 Liverneaux $\mathrm{P}$, Nectoux $\mathrm{E}$, Taleb C. The future of robotics in hand surgery. Chir Main 2009;28:278-85.

80 Lundborg G, Rosen B. Hand function after nerve repair. Acta Physiol 2007;189:207-17.

81 Oud T, Beelen A, Eijffinger $E$, et al. Sensory re-education after nerve injury of the upper limb: a systematic review. Clin Rehabil 2007;21:483-94.

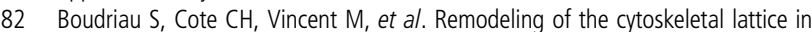
denervated skeletal muscle. Muscle Nerve 1996;19:1383-90.

83 Borisov AB, Dedkov El, Carlson BM. Interrelations of myogenic response, progressive atrophy of muscle fibers, and cell death in denervated skeletal muscle. Anat Rec 2001;264:203-18.

84 Fu SY, Gordon T. Contributing factors to poor functional recovery after delayed nerve repair: prolonged denervation. J Neurosci 1995;15(5 Pt 2):3886-95.

85 Fu SY, Gordon T. Contributing factors to poor functional 1 recovery after delayed nerve repair: prolonged axotomy. J Neurosci 1995;15(5 Pt 2):3876-85.

86 Brushart TM, Tarlov EC, Mesulam MM. Specificity of muscle reinnervation after epineurial and individual fascicular suture of the rat sciatic nerve. J Hand Surg 1983;8:248-53.

87 Al-Majed AA, Brushart TM, Gordon T. Electrical stimulation accelerates and increases expression of BDNF and trkB mRNA in regenerating rat femoral motoneurons. Eur J Neurosci 2000;12:4381-90.

88 Al-Majed AA, Neumann CM, Brushart TM, et al. Brief electrical stimulation promotes the speed and accuracy of motor axonal regeneration. J Neurosci 2000:20:2602-8.

89 Gordon T, Chan KM, Sulaiman OA, et al. Accelerating axon growth to overcome limitations in functional recovery after peripheral nerve injury. Neurosurgery 2009:65(4 Suppl):A132-44

90 Cobianchi S, Casals-Diaz L, Jaramillo J, et al. Differential effects of activity dependent treatments on axonal regeneration and neuropathic pain after peripheral nerve injury. Exp Neurol 2013;240:157-67. 
91 Udina E, Puigdemasa A, Navarro X. Passive and active exercise improve regeneration and muscle reinnervation after peripheral nerve injury in the rat. Muscle Nerve 2011:43:500-9.

92 Lewin-Kowalik J, Marcol W, Kotulska K, et al. Prevention and management of painful neuroma. Neurol Med Chir (Tokyo) 2006;46:62-8.

93 Souza JM, Cheesborough JE, Ko JH, et al. Targeted muscle reinnervation: a novel approach to postamputation neuroma pain. Clin Orthop Relat Res 2014:472:2984-90.

94 Richardson RR, Meyer PR, Cerullo LJ. Neurostimulation in the modulation of intractable paraplegic and traumatic neuroma pains. Pain 1980;8:75-84.

95 Leung A, Fallah A, Shukla S. Transcutaneous magnetic stimulation (TMS) in alleviating post-traumatic peripheral neuropathic pain States: a case series. Pain Med 2014;15:1196-9.

96 Rafuse VF, Gordon T, Orozco R. Proportional enlargement of motor units after partial denervation of cat triceps surae muscles. J Neurophysiol 1992;68:1261-76.

97 Valero-Cabre A, Tsironis K, Skouras E, et al. Peripheral and spinal motor reorganization after nerve injury and repair. J Neurotrauma 2004;21:95-108.

98 Navarro X, Vivo M, Valero-Cabre A. Neural plasticity after peripheral nerve injury and regeneration. Prog Neurobio/ 2007;82:163-201.

99 Scarfone H, McComas AJ, Pape K, et al. Denervation and reinnervation in congenital brachial palsy. Muscle Nerve 1999;22:600-7.

100 Brown T, Cupido C, Scarfone $H$, et al. Developmental apraxia arising from neonatal brachial plexus palsy. Neurology 2000;55:24-30.

101 Colon AJ, Vredeveld JW, Blaauw G. Motor evoked potentials after transcranial magnetic stimulation support hypothesis of coexisting central mechanism in obstetric brachial palsy. J Clin Neurophysiol 2007;24:48-51.
102 Korak KJ, Tam SL, Gordon T, et al. Changes in spinal cord architecture after brachial plexus injury in the newborn. Brain 2004;127(Pt 7):1488-95.

103 Chen R, Cohen LG, Hallett M. Nervous system reorganization following injury. Neuroscience 2002;111:761-73.

104 Rossini PM, Martino G, Narici L, et al. Short-term brain 'plasticity' in humans: transient finger representation changes in sensory cortex somatotopy following ischemic anesthesia. Brain Res 1994;642:169-77.

105 Qiu TM, Chen L, Mao Y, et al. Sensorimotor cortical changes assessed with resting-state fMRI following total brachial plexus root avulsion. J Neurol Neurosurg Psychiatry 2014;85:99-105.

106 Wiech K, Preissl H, Lutzenberger W, et al. Cortical reorganization after digit-to-hand replantation. J Neurosurg 2000;93:876-83.

107 Mano Y, Nakamuro T, Tamura R, et al. Central motor reorganization after anastomosis of the musculocutaneous and intercostal nerves following cervical root avulsion. Ann Neurol 1995;38:15-20.

108 Pascual-Leone A, Torres F. Plasticity of the sensorimotor cortex representation of the reading finger in Braille readers. Brain 1993;116(Pt 1):39-52.

109 Charlton CS, Ridding MC, Thompson PD, et al. Prolonged peripheral nerve stimulation induces persistent changes in excitability of human motor cortex. J Neurol Sci 2003;208:79-85.

110 Rosenkranz K, Rothwell JC. Differences between the effects of three plasticity inducing protocols on the organization of the human motor cortex. Eur J Neurosci 2006;23:822-9.

111 Lefaucheur JP, Antal A, Ahdab R, et al. The use of repetitive transcranial magnetic stimulation (rTMS) and transcranial direct current stimulation (tDCS) to relieve pain Brain Stim 2008;1:337-44. 